\title{
Moringa oleifera leaf extract ameliorated high-fat diet-induced obesity, oxidative stress and disrupted metabolic hormones
}

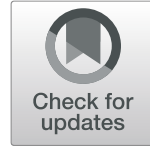

\author{
Azza I. Othman', Maher A. Amer², Asmaa S. Basos and Mohammed A. El-Missiry ${ }^{1 *}$ [D
}

\begin{abstract}
Background: Obesity is a health problem in many countries, and maintaining a perfect weight is challenging. Moringa oleifera leaf extract (ME) is rich in polyphenols with antioxidant and pharmaceutical potential. The present study investigated the potential protective effect of Moringa oleifera leaf extract against obesity induced from a high-fat diet (HFD), oxidative stress and disruption of metabolic hormones compared to simvastatin (SIM) or their combination.

Results: Rats fed a HFD for 6 weeks exhibited a significant increase in body weight and levels of serum glucose and lipid fractions, verifying an obesity state. There were also higher levels of insulin and leptin and lower gherlin in sera of HFD rats compared to the levels in control rats. Homeostasis model assessment for insulin resistance (HOMA-IR), quantitative insulin sensitivity check index (QUICKI) and the atherogenic index were elevated, indicating the development of insulin resistance and dyslipidaemia in these rats. These changes were accompanied with a significant increase in oxidative stress, as indicated by elevated lipid peroxidation and protein oxidation with low levels of antioxidants in liver. The activities of liver function enzymes, including aspartate amino transferase, alanine amino transferase, alkaline phosphatase and gamma glutamyltransferase, were also significantly increased in serum. Concurrent treatment with $300 \mathrm{mg} / \mathrm{kg} \mathrm{ME}$ for 6 weeks ameliorated the increase in body weight and improved the levels of glucose, lipid fractions and metabolic hormones, indicating the anti-obesity effect and amelioration of tissue insulin resistance potential of ME. ME treatment also normalized oxidative stress and antioxidants in liver and improved liver function enzymes, indicating the antioxidant potential of ME. The effects of ME were similar to SIM, and the combination of these agents was better than each agent alone.
\end{abstract}

Conclusion: We propose that ME extract has anti-obesity and antioxidant potential and may be used as a lipidlowering drug to control weight, obesity and its pathophysiological consequences.

Keywords: Obesity, Polyphenols, Insulin, Leptin, Gherlin, Antioxidants, Oxidative stress, Liver

\section{Introduction}

Complementary and alternative medicine is used worldwide for the treatment of a variety of diseases and disorders that involve oxidative stress. Plant polyphenols are effective antioxidants that are used to control oxidative stress in different diseases. Plant polyphenols scavenge free radicals, chelate transition metals, such as iron and copper, and protect endogenous antioxidant systems [1]. These polyphenols are found in all plant parts and constitute an important part of the human diet. The main

\footnotetext{
*Correspondence: elmissiry@mans.edu.eg; malemissiry@yahoo.com ${ }^{1}$ Zoology Department, Faculty of science, Mansoura University, Mansoura, Egypt

Full list of author information is available at the end of the article
}

source of natural polyphenols is medicinal plants, which differ widely in functional group and biological activity in the management of human diseases [2].

Many plant extracts and their purified compounds control blood glucose and reverse physiological abnormalities [3]. It is crucial that agents that decrease oxidative stress be therapeutically beneficial. Therefore, the anti-obesity and antioxidant properties of plant polyphenols are linked. Oxidative stress markers are increased in obese subjects. An increase in reactive oxygen species (ROS) production in adipose tissue is associated with the upregulation of NADPH oxidase and the downregulation of antioxidant enzymes [4]. Rats fed a high-fat diet (HFD) gained body weight with increased oxidative stress in liver 
[5] and muscle [6]. The latter author reported an increase in the production of hydrogen peroxide and a decrease in superoxide dismutase in muscle. HFD also results in severe changes in mitochondrial lipids and overproduction of reactive oxygen species (ROS) in liver [7].

The consumption of a high-fat diet is related to metabolic syndromes, particularly diabetes $[8]$ and obesity $[9,10]$. These disorders increase the risk of developing insulin resistance and hypertension [11]. Insulin resistance refers to an insufficient response to normal levels of insulin in peripheral target tissue, including liver, muscle and adipose tissue. Despite the advances of new medicine, diabetes and obesity remain the leading cause of morbidity and mortality, with increasing social and health disruption, worldwide [8]. Therefore, natural and safe agents that reduce obesity, rather than surgical intervention and synthetic drug application, are an important issue in modernized and developing countries.

Insulin, leptin and gherlin are the three metabolic hormones responsible for the management of blood glucose level, regulation of body fat stores, energy expenditure and body weight gain [12]. The type and amount of food intake markedly affect the levels and balance between these hormones. Disruption of these hormones significantly impacts the development of metabolic syndrome in obese patients [13].

Moringa oleifera Lam is a small-sized tree that belongs to the family Moringaceae, and it is cultivated in Africa and Asia. The leaves of $M$ oleifera exhibit pharmaceutical activity due to its phytoconstituents and natural antioxidant molecules, including vitamins and carotenoids $[14,15]$. The beneficial pharmacological actions of $M$ oleifera include anti-inflammatory, wound-healing, diuretic [16], antifungal [17] and antioxidant [18] activities.

Simvastatin is marketed as Zocor, and it belongs to the statin pharmaceutical group, which are used to lower blood cholesterol in different conditions [19]. Simvastatin showed significant protective effects on endothelial cells and anticoagulation [20]. Simvastatin unfortunately also has numerous adverse side effects, such as gastrointestinal disorders, headache, and rash [21], and some of these effects are a real threat to the life and health of patients [22].

An increasing number of studies reported the hypoglycaemic and antioxidant effects of Moringa oleifera $[14,15,18]$. The present study investigated the potential protective effect of Moringa oleifera leaf extract against HFD-induced obesity, oxidative stress and the disruption of metabolic hormones in HFD fed rats compared to the cholesterol-lowering drug simvastatin and their combination.

\section{Materials and methods}

\section{Chemicals}

Simvastatin was obtained from Global Napi Pharmaceuticals, Egypt. Cholesterol was purchased from Sigma chemical company (St. Louis, MO, USA). All other chemicals were of analytical grade.

\section{Preparation of Moringa oleifera leaf extract}

Leaves of Moringa oleifera were obtained from a local market in Mansoura and authenticated in the Botany Department of Mansoura University Egypt. The leaves were cleaned, dried and ground. The ground leaves (40 g) were macerated in ethyl alcohol (70\%) with continuous stirring for 3 days at $4{ }^{\circ} \mathrm{C}$. The extract was filtered and lyophilized to obtain a solid powder, which was stored at $4{ }^{\circ} \mathrm{C}$. The yield of the extract was $17.5 \%$. The dose used in the current experiment was $300 \mathrm{mg} / \mathrm{kg}$ and prepared in distilled water for oral administration [23].

\section{Animals}

Adult male Wistar rats, 200-230 g, were obtained from the Holding Company for Biological Products and Vaccines (VACSERA, Cairo, Egypt). The animals were placed in stainless steel cages under normal laboratory conditions and exposed to 12-h light/dark cycles. All rats were acclimated for one week before experimentation. The experimental protocol was performed in accordance with the guidelines of the National Institutes of Health for the Care and Use of Laboratory Animals (NIH Publication No. 8523, revised 1996) and conformed to the local experimental animal ethics committee of Mansoura University, Egypt.

\section{Animal groups}

Animals were divided into six groups of six rats. Control group rats received an ordinary diet and water ad libitum. In group 2, the Moringa leaf extract (ME)-treated group received daily oral administration of $300 \mathrm{mg} / \mathrm{kg}$ [23] body weight for six weeks. Rats in the 3rd group received an HFD daily for six weeks. Rats in the 4th group were fed an HFD and concomitantly received oral ME extract daily for six weeks. The 5th group received HFD and simvastatin (SIM) $40 \mathrm{mg} / \mathrm{kg}$ daily for six weeks. SIM administered by gavage. Rats in the 6th group received an HFD and ME and SIM at the same doses and rout of administration as the other groups. The HFD was a mixture of $68 \%$ standard chow diet, $30 \%$ animal fats and $2 \%$ cholesterol [24].

Body weights were recorded on the first day of the experiment then weekly for consecutive six weeks using a digital weighing balance.

Rats were fasted overnight after six weeks and anaesthetized using Ketamine and xylazine mixture. Blood samples were collect via cardiac puncture. Serum samples were obtained after centrifugation at $5000 \mathrm{x}$ g for $10 \mathrm{~min}$ at $4{ }^{\circ} \mathrm{C}$. Anaesthetized rats were euthanized and dissected to obtain liver samples, which were cleaned and homogenized in chilled Tris- $\mathrm{HCl}$ buffer $(0.1 \mathrm{M}) \mathrm{pH}$ 
7.4 in Potter-Elvehjem Tissue Grinders for biochemical analyses.

\section{Biochemical analyses}

Levels of serum glucose and lipid fractions (triglycerides (TG), total cholesterol (TC) and high-density lipoprotein (HDL cholesterol)) were assayed using a colorimetric assay kit from Biodiagnostic Co, Egypt. The value of LDL was estimated using the eq. $\mathrm{LDL}=$ cholesterol - triglycerides/5 -HDL.

Serum insulin, leptin, gherlin, T3 and T4 levels were estimated using rat ELISA kits (Siemens healthcare diagnostics, Cambridge, MA, USA). The homeostasis model assessment for insulin resistance (HOMA-IR), quantitative insulin sensitivity check index (QUICKI) and atherogenic index were estimated as previously described [25].

Activities of aspartate amino transferase, alanine amino transferase, alkaline phosphatase and gamma glutamyltransferase in sera were measured using a kit from Elitech Group, Puteaux, France.

Malondialdehyde (MDA) was measured using the reaction of MDA with thiobarbituric acid to yield thiobarbituric acid reactive substances (TBARS) following the manufacturer's instructions (Biodiagnositic Kit, Egypt). Protein carbonyl level was estimated following the instructions of a colorimetric assay kit from Cayman Chemical, USA. The Nitric oxide was estimated using Biodiagnositic Kit, Egypt. Total antioxidant capacity, glutathione (GSH) level and superoxide dismutase (SOD), catalase (CAT) glutathione peroxidase (GPx), and glutathione reductase (GR) activities were determined as described in the Biodiagnositic Kit, Egypt.

\section{Statistical analyses}

Data were analysed using GraphPad Prism 5.0 (GraphPad Software Inc., San Diego, CA, USA), and the results are expressed as the means $\pm \mathrm{SD}(n=6)$. Statistical comparisons were performed using one-way ANOVA followed by Student's Newman-Keuls post-hoc test. A significant difference was considered at $P<0.05$.
Different letters indicate significant differences between groups $(p<0.05)$. Values represent means $\pm \mathrm{SD}(N=6)$.

\section{Results}

HFD rats exhibited a marked increase in body weight compared to normal diet animals (Table 1). Two weeks of Moringa leaf extract (ME) treatment markedly reduced the percentage of body weight gain compared to HFD supplemented rats. Subsequent body weight gain at the 3rd, 4th, 5th and 6th weeks were lower than the HFD group. Rats fed simvastatin (SIM) or ME plus SIM exhibited lower body weight gain than rats fed an HFD.

The effects of ME and SIM on lipids in HFD rats were investigated (Fig. 1). Animals fed an HFD for six consecutive weeks showed significant increases in total lipids, cholesterol, triglycerides and LDL, reduced HDL (Fig. 1) and increased atherogenic index (Fig. 1) compared to control rats fed a normal diet. Treatment with ME, SIM or both agents significantly prevented the dyslipidaemia and reduced atherogenic index in HFD rats. Similar results were obtained using the standard drug SIM and SIM plus ME on lipids and the atherogenic index.

The HFD fed rats showed a significant increase in serum levels of glucose, and leptin and decreased levels of insulin and gherlin (Fig. 2). A significant increase in HOMA-IR and significant decrease in QUICKI compared to control animals was also observed (Fig. 2). Concurrent supplementation with ME prevented hyperglycaemia and ameliorated the changes in insulin, gherlin and leptin levels. Similar effects were observed after treatment with SIM and the combination of SIM and ME. Treatment of HFD rats with the combination of ME and SIM for 6 weeks produced superior beneficial effects on insulin and leptin compared to each agent alone.

Rats fed HFD showed a significant decrease in blood levels of T3 and T4 compared to the control group (Fig. 3). Treatment with ME, SIM and their combination significantly ameliorated these changes and showed comparable values to the control group.

Table 1 The effects of Moringa leaf extract (ME), simvastatin (SIM) and their combination (ME+SIM) on body weight gain in rats fed a high-fat diet (HFD) for 6 weeks. ME controlled body weight gain and ameliorated obesity. All values are means \pm SD $(n=6)$

\begin{tabular}{|c|c|c|c|c|c|c|}
\hline & Control & ME & HFD & $\mathrm{HFD}+\mathrm{ME}$ & $\mathrm{HFD}+\mathrm{SIM}$ & $\mathrm{HFD}+\mathrm{ME}+\mathrm{SIM}$ \\
\hline Initial weight & $214.5 \pm 2.43$ & $219.5 \pm 3.31$ & $216.5 \pm 7.1$ & $214.7 \pm 5.79$ & $218.7 \pm 3.09$ & $215.2 \pm 3.60$ \\
\hline 1st week & $224.8 \pm 3.24$ & $221.3 \pm 1.92$ & $238.7 \pm 10.06$ & $224.5 \pm 5.48$ & $220.2 \pm 6.36$ & $217.3 \pm 4.37$ \\
\hline 2nd week & $231.3 \pm 3.57$ & $237.5 \pm 4.03$ & $255 \pm 9.95$ & $233.8 \pm 9.75$ & $236 \pm 12.95$ & $236.3 \pm 7.09$ \\
\hline 3rd week & $242.7 \pm 4.78$ & $241 \pm 5.28$ & $288.2 \pm 23.44$ & $249.3 \pm 10.28$ & $250.2 \pm 7.43$ & $238.5 \pm 4.19$ \\
\hline 4th week & $245 \pm 7.73$ & $252.8 \pm 7.56$ & $297.2 \pm 23.5$ & $283.3 \pm 8.44$ & $271.7 \pm 13.94$ & $260.8 \pm 5.83$ \\
\hline 5th week & $254.7 \pm 7.09$ & $257.3 \pm 4.24$ & $304.3 \pm 5.90$ & $283.3 \pm 3.66$ & $274.2 \pm 15.02$ & $261 \pm 10.94$ \\
\hline 6th week & $265.0 \pm 7.05$ & $264.3 \pm 4.06$ & $317.7 \pm 6.62$ & $268.3 \pm 9.14$ & $270.8 \pm 7.60$ & $266.0 \pm 5.47$ \\
\hline$\%$ of change from initial weight & 23.5 & 20 & 38 & 24.9 & 23.8 & 23.6 \\
\hline
\end{tabular}




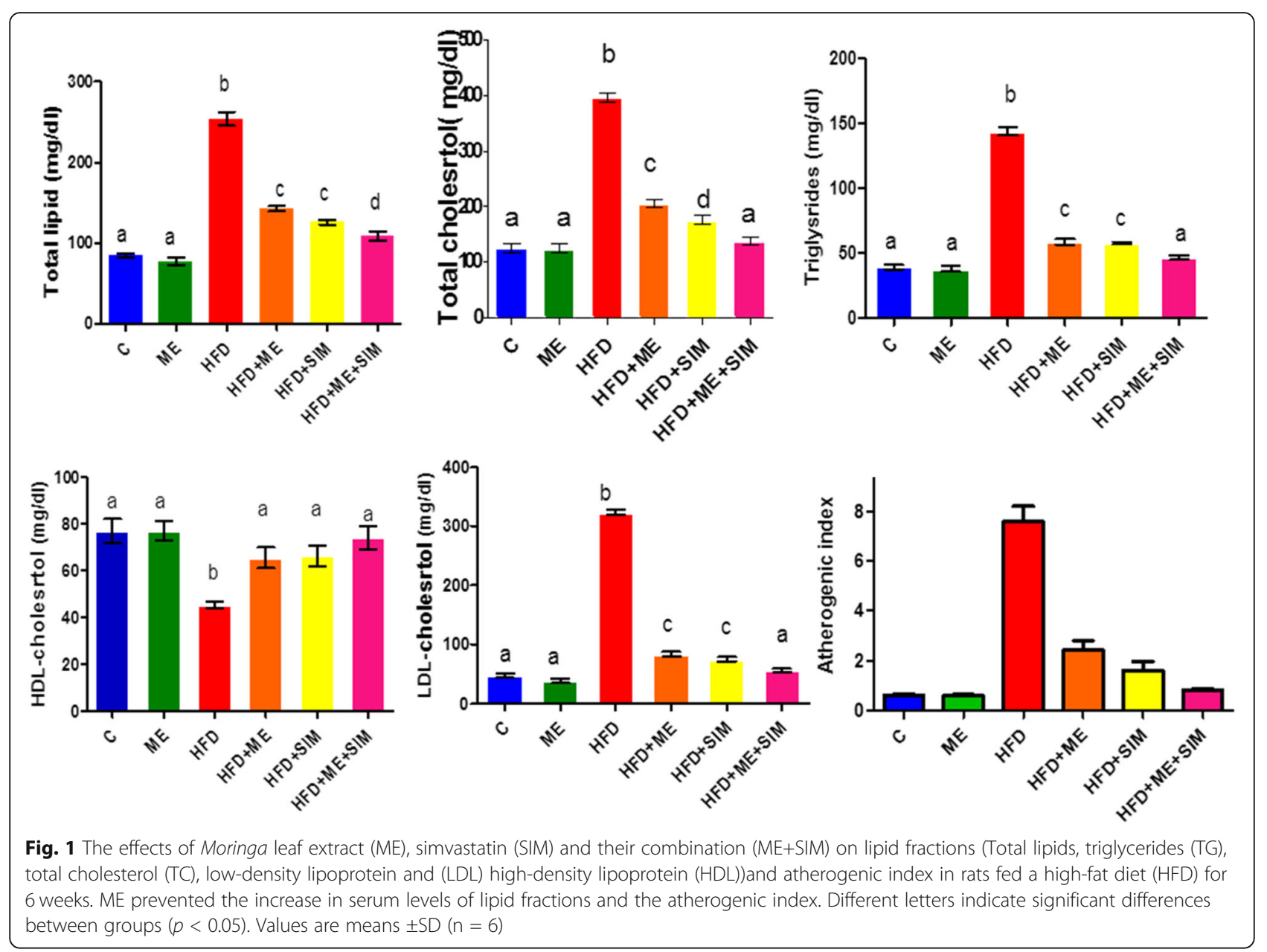

Figure 4 shows that rats fed HFD for 6 weeks showed significant elevation in liver enzymes, including ALT, AST, AP and GGT, compared to the control group. Treatment with ME concurrently with HFD significantly ameliorated the increase in liver enzymes. Similar results were obtained using the standard drug SIM and the combination of SIM and ME.

HFD rats exhibited marked increases in lipid peroxidation, protein oxidation and nitric oxide (Fig. 5) and decreased levels of GSH and GR, GPx, SOD and CAT activities in liver (Fig. 6). Treatment with ME, SIM or their combination resulted in a significant prevention of these changes in oxidative stress parameters and antioxidants in the liver. The combination of SIM and ME exerted a superior impact than each drug alone (Fig. 5).

\section{Discussion}

Obesity is a worldwide health problem, and it is considered the major risk factor for morbidity and mortality. High-fat diet (HFD) intake is responsible for body weight gain and obesity because of its high energy and fat contents. The present results demonstrated a significant body weight gain in rats fed an HFD for six weeks, which indicated an obesity state. This obese model is consistent with several previous studies $[11,26]$. To prevent obesity and related disorders, a suggested promising strategy is the use plant extracts that contain a mixture of polyphenols. The current study revealed that $\mathrm{ME}$ reduced body weight gain, prevented the increase in cholesterol, LDL and TG and increased HDL compared to HFD-supplemented rats. Similar effects were observed using simvastatin (SIM) alone and in combination with ME. This result confirms a previous study using methyl extract of moringa leaves, which remarkably decreased body and organ weights and indicated the antiobesity effect of ME [21]. The present data are consistent with a report in which treatment with a crude extract of ME increased blood HDL level and lowered levels of cholesterol, LDL and triglycerides [27]. These results indicate that ME is a useful agent to ameliorate dyslipidaemia and the atherogenic impact and possibly provide protection from hypertension and cardiovascular diseases.

The present study revealed that HFD increased serum glucose, insulin and leptin levels and increased HOMA-IR, which were significantly ameliorated by the concurrent 

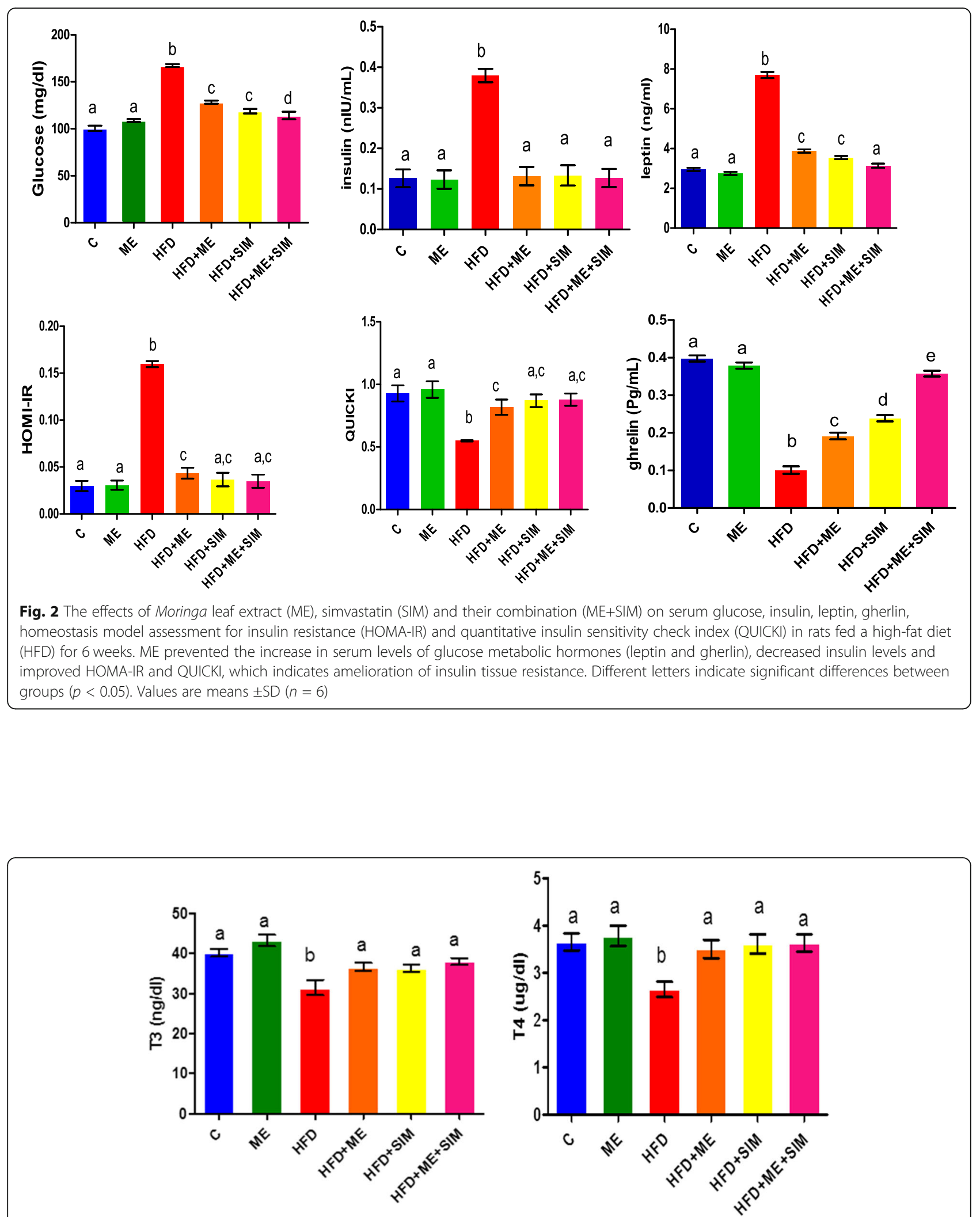

Fig. 3 The effects of Moringa leaf extract (ME), simvastatin (SIM) and their combination (ME+SIM) on serum T3 and T4in rats fed a high-fat diet (HFD) for 6 weeks. ME prevented the decrease in the serum levels of these hormones. Different letters indicate significant differences between groups $(p<0.05)$. Values are means $\pm S D(n=6)$ 

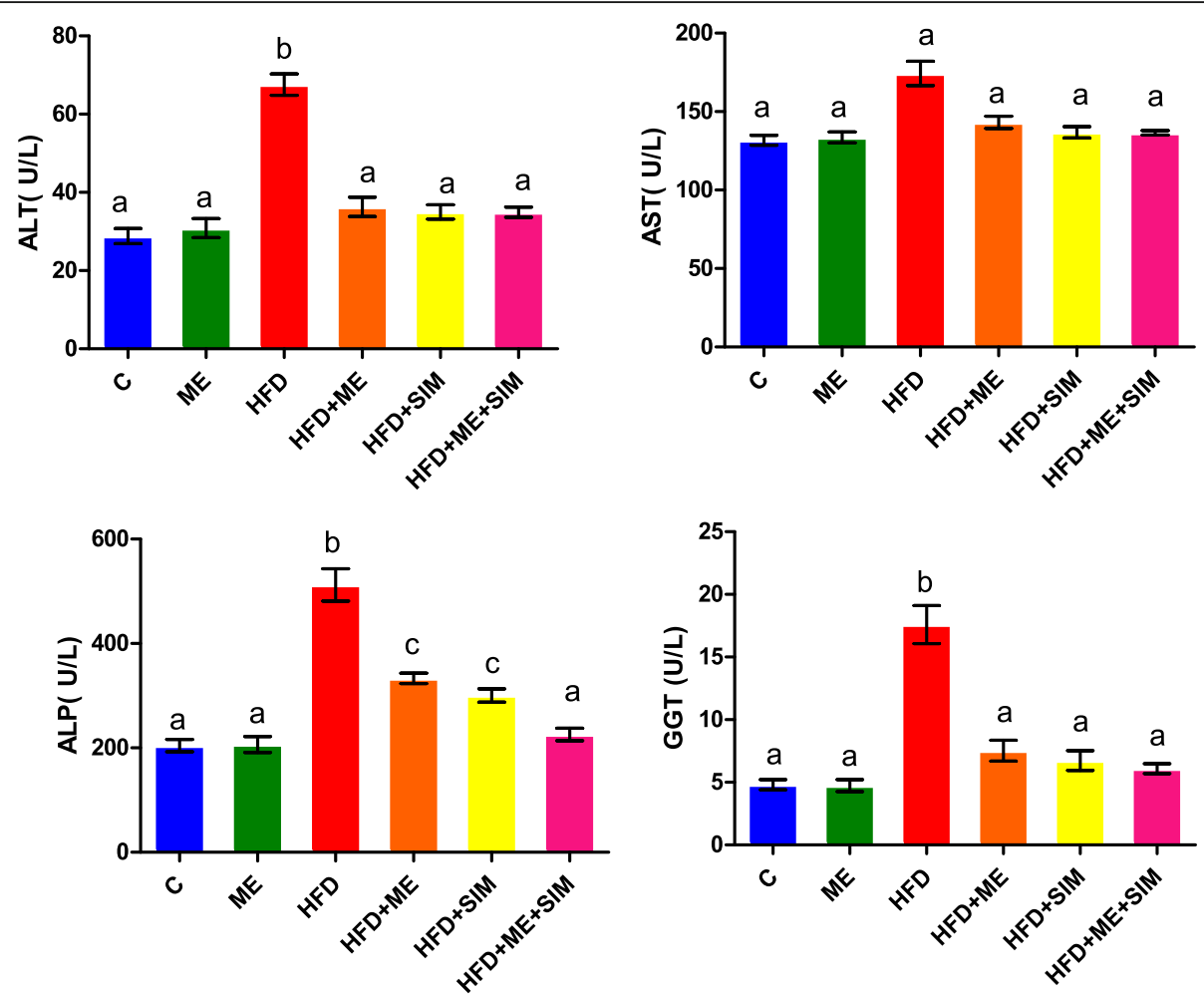

Fig. 4 The effects of Moringa leaf extract (ME), simvastatin (SIM) and their combination (ME+SIM) on the activities of serum aspartate amino transferase (AST), alanine amino transferase (ALT), alkaline phosphatase (ALP) and gamma glutamyltransferase (GGT) in rats fed a high-fat diet (HFD) for 6 weeks. ME protected liver tissue, which was indicated by the maintenance of comparable activities to the control. Different letters indicate significant differences between groups $(p<0.05)$. Values are means $\pm S D(n=6)$

supplementation of ME, SIM and their combination compared to control rats. ME showed similar effects to SIM and their combination. ME improved tissue insulin resistance, glucose levels and reduced body weight gain in HFD fed rats, which supports previous studies using polyphenol-rich plant extracts [28, 29]. Insulin and glucose regulate leptin levels. Previous studies showed a positive correlation between leptin and body fats regulation by insulin [30] and glucose metabolism [31]. Elevated leptin reflected insulin resistance [32] and showed a significant correlation with HOMA-IR [13].

The current study demonstrated that gherlin secretion was significantly decrease in HFD fed rats. The mechanism of reduced gherlin secretion is not clear. There are four proposed factors to explain the suppression of gherlin levels, including hyperinsulinemia [33], hyperglycaemia [34], hyperleptinemia [35] and elevated body weight [36]. The current study supports these possibilities based on increased insulin, leptin, and glucose levels and elevated body weights in HFD fed rats. Suppression of gherlin secretion from the rat stomach is linked to higher gastric somatostatin release [37]. These results indicate that several hormonal signals control gherlin secretion from the stomach. Treatment with ME, SIM and their combination improved gherlin levels, and the combined treatment was superior in the upregulation of gherlin levels in HFD fed rats.

The present study demonstrated low levels of T3 and T4 in rats fed HFD, which indicates hypothyroxinemia. This finding supports a recent report that increased intake of an HFD and increased serum lipid fractions caused disturbances in the thyroid lipid profile and hypothyroxinemia and increased TSH levels in response to decreased thyroid hormones [38], which was ameliorated by dietary modification [39]. Maintaining thyroid homeostasis is essential for human health. Concurrent intake of ME corrected the disturbance in thyroid hormones likely via the hypothalamus-pituitary-thyroid axis [40].

Obesity is associated with increased oxidative stress due to increased free radical production and disturbed antioxidant capacity, particularly the inhibition of antioxidant enzymes [41]. There is an increase in oxidative stress in adipose tissue and livers of mice fed an HFD [42]. Early expression of genes related to ROS production is enhanced in HFD fed rats [26]. Obesity associated with increased leptin levels promoted inflammatory reactions with an increase in cytokine activation of 

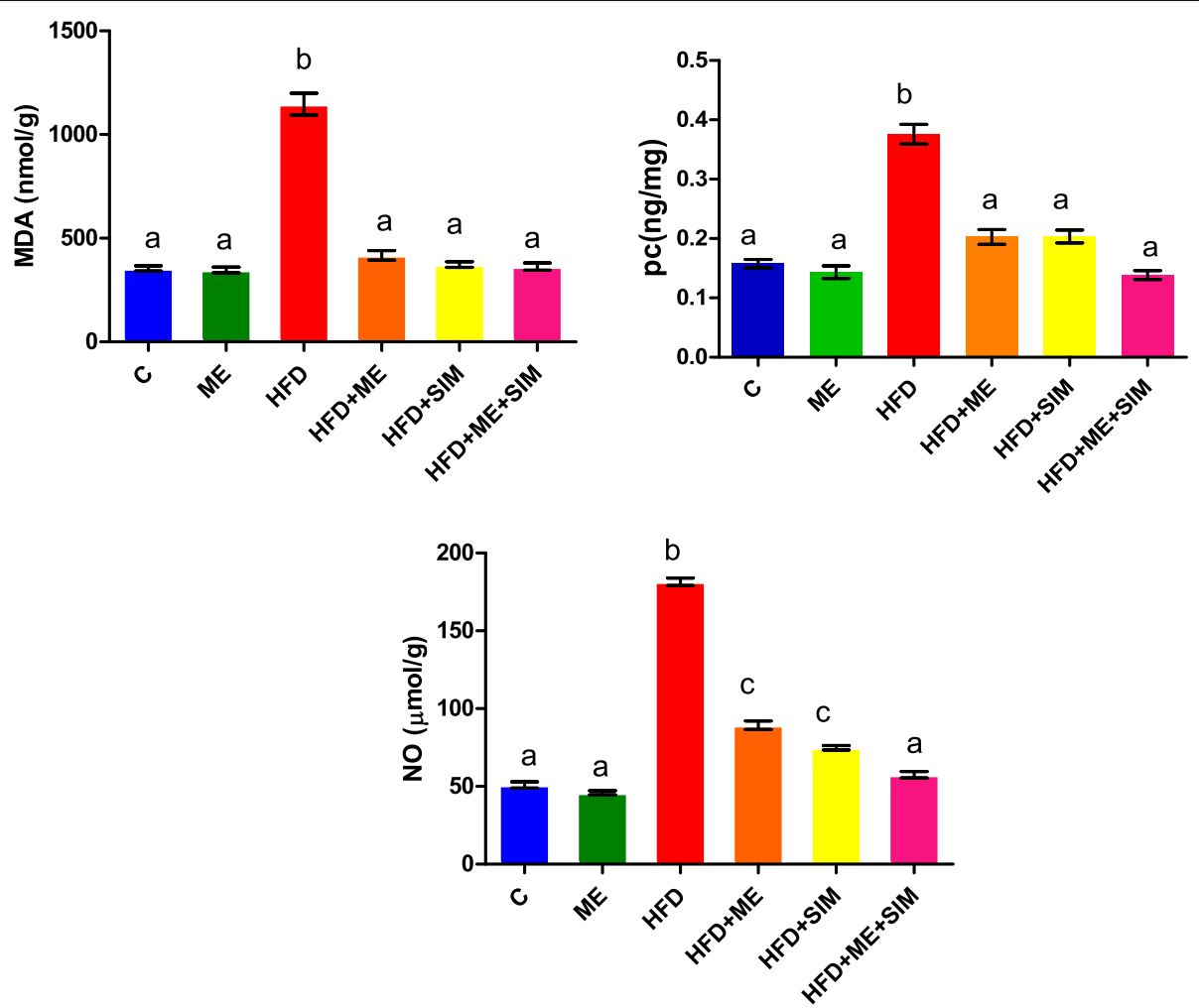

Fig. 5 The effects of Moringa leaf extract (ME), simvastatin (SIM) and their combination (ME+SIM) on the levels of lipid peroxidation (as indicated by malondialdehyde (MDA)), protein oxidation (represented by protein carbonyl $(\mathrm{PO})$ ) and nitric oxide $(\mathrm{NO})$ in the livers of rats fed a high-fat diet (HFD) for 6 weeks. ME protected liver tissue as indicated by the maintenance of comparable activities to the control. Different letters indicate significant differences between groups $(p<0.05)$. Values are means $\pm \operatorname{SD}(n=6)$

NADPH oxidase, which increased the ROS production that oxidized lipids and proteins [43,44]. The present study confirmed these studies and demonstrated a significant increase in lipid peroxidation and protein oxidation and decreased levels of antioxidants in the liver of rats fed HFD for six weeks. Treatment with ME alone and in combination with SIM ameliorated the oxidative stress in liver, and likely other body organs, likely due to polyphenolic compounds, which may scavenge and inhibit ROS overproduction. These data suggest that the use of polyphenol-rich plant extracts exhibit anti-obesity and antioxidant functions to overcome the adverse consequences of a high-calorie diet. Plants rich in phenolic compounds may be used as dietary antioxidants to prevent oxidative stress induced-damage [45]. The current study confirms this suggestion and showed that ME protected against HFD-induced oxidative stress and maintained remarkable levels of antioxidants in the liver. This result indicates that ME suppresses the development of endogenous oxidative stress in liver, which improves insulin resistance, hyperglycaemia and leptin levels. Insulin resistance is a consequence of the consumption of an HFD and increased production of ROS [46].
ME treatment normalized several antioxidants in the livers of rats fed HFD. Superoxide dismutase (SOD) and catalase (CAT) are two antioxidant enzymes that are responsible for the dismutation of superoxide anions into hydrogen peroxide, water and oxygen. SOD eliminates superoxide radicals in liver and decreases blood glucose levels [47]. Concurrent treatment with ME produced higher activities of SOD and CAT in the livers of HFD fed rats, which indicates the ability of ME to scavenge ROS or protect the SOD/CAT system that eliminated superoxide radicals. An important factor to determine changes in the redox balance in the body is the total antioxidant capacity (TAC), which expresses the main scavenging capability [48]. The current study demonstrated that ME enhanced TAC in the livers of HFD fed rats, which indicates the anti-oxidative ability of ME.

The protection observed after ME treatment of HFD fed rats suggested a prophylactic impact of ME against HFD-induced metabolic disruption via the normalization of ROS. ME upregulated glutathione (GSH) in the livers of HFD fed rats compared to control rats in the present study. GSH is important in the elimination of free 

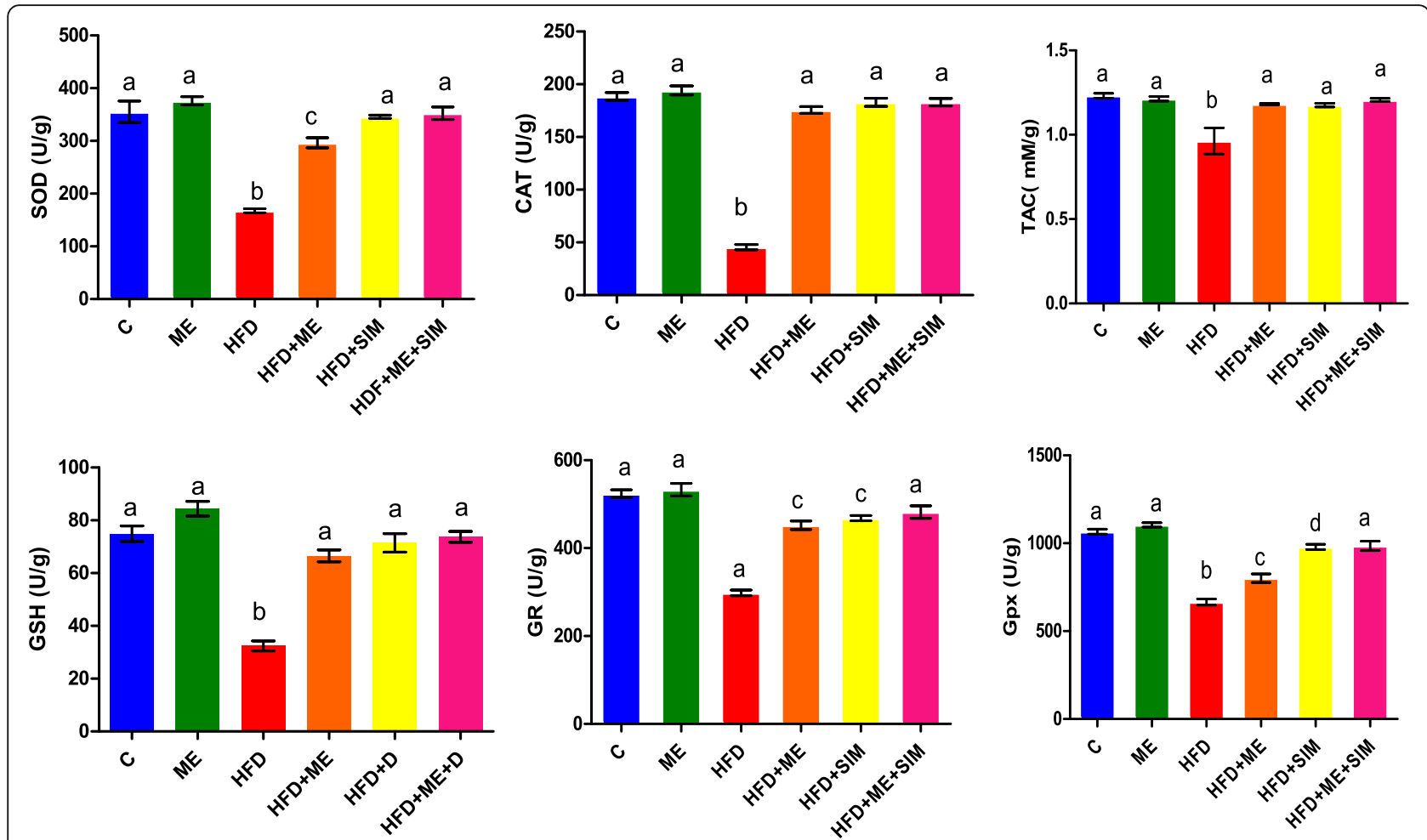

Fig. 6 The effects of Moringa leaf extract (ME), simvastatin (SIM) and their combination (ME+SIM) on glutathione (GSH) concentration, superoxide dismutase (SOD), catalase (CAT), glutathione reductase, and glutathione peroxidase activities and total antioxidant capacity in livers of rats fed a high-fat diet (HFD) for 6 weeks. ME protected liver tissue as indicated by the maintenance of comparable activities to the control. Different letters indicate significant differences between groups $(p<0.05)$. Values are means $\pm S D(n=6)$

radicals, and it acts as substrate for glutathione peroxidase (GPx) to neutralize hydrogen peroxide and organic hydroperoxides in lipid cellular membranes against oxidative damage [49]. Increasing activity of GPx remarkably reduces oxidative stress in liver [50]. The antioxidative effect of ME on lipid and protein oxidation may be related to the phenolic compounds of this plant extract. Notably, the ratio between reduced and oxidized glutathione (GSH/GSSG) plays a key role in glucose homeostasis in obesity because sulphhydryl groups are crucial in the hepatocyte redox state [51]. ME may have upregulated GSH content via improving glutathione reductase (GR) activity, which reduced the GSSG to GSH and improved the GSH/GSSG ratio. These changes consequently decreased lipid and protein oxidation and improved glucose and its metabolism.

HFD impairs nitric oxide (NO) stability and synthesis and induces insulin resistance [52]. NO is extensively produced by endothelial cells, Kupffer cells and hepatocytes in response to inflammation [53]. Excessive production of $\mathrm{NO}$ is cytotoxic due to the production of hydroxyl radicals and may induce oxidative stress [54]. The present study demonstrated high levels of NO in HFD fed rats. This elevation was significantly ameliorated after treatment with ME, SIM and their combination. Low NO production enhances glucose metabolism via the upregulation of skeletal muscle glucose uptake through the Glut transporter and increases and activates mitochondrial biogenesis [55]. This effect may be the potential protective mechanism on ME in the livers of obese subjects.

The liver is a multifunctional organ that is responsible for whole body metabolism, and it is subjected to increased production of ROS and oxidative stress damage [53]. This role exacerbates the induction of metabolic syndrome and liver damage. Suppression of oxidative stress and the upregulation of systemic antioxidants by ME may play important roles in liver protection during consumption of an HFD. ME protected the livers of mice fed an HFD, which was indicated by the normalization of liver function enzymes, including ALT, AST, AP and GGT.

In conclusion, Moringa leaf extract controlled body weight gain when given concurrently with HFD, which indicates a lower obesity state. This effect was accompanied with improvements in serum lipid fractions, glucose, metabolic hormones and lower oxidative stress and liver function. The underlying mechanism for the ameliorative changes related to obesity may be attributed to the antioxidant effects of the polyphenols in ME. Improving redox balance pathways is a good therapeutic strategy in cases of obesity and its related adverse consequences. 


\section{Acknowledgments}

Facilities provided by Faculty of Science, Mansoura University, Egypt, are greatly acknowledged and appreciated.

\section{Authors' contributions}

All authors contributed equally. All authors read and approved the final manuscript.

\section{Funding}

Authors declare that they did not receive any fund.

\section{Availability of data and materials}

The datasets used and/or analysed during the current study are available from the corresponding author on reasonable request.

\section{Ethics approval and consent to participate}

The experimental protocol was performed in accordance with the guidelines of the National Institutes of Health for the Care and Use of Laboratory Animals (NIH Publication No. 8523, revised 1996) and conformed to the local experimental animal ethics committee of Mansoura University, Egypt.

\section{Consent for publication}

Not applicable

\section{Competing interests}

All authors declare that they have no conflict interests.

\section{Author details}

'Zoology Department, Faculty of science, Mansoura University, Mansoura, Egypt. ${ }^{2}$ Zoology Department, Faculty of science, Azzytuna University, Tarhuna, Libya.

Received: 5 August 2019 Accepted: 11 December 2019 Published online: 26 December 2019

\section{References}

1. Dai J, Mumper RJ. Plant phenolics: extraction, analysis and their antioxidant and anticancer properties. Molecules. 2010;15(10):7313-52.

2. Saravanan G, Ponmurugan $P$, Sathiyavathi M, Vadivukkarasi S, Sengottuvelu S. Cardioprotective activity of Amaranthus viridis Linn: effect on serum marker enzymes, cardiac troponin and antioxidant system in experimental myocardial infarcted rats. Int J Cardiol. 2013;165(3):494-8.. Epub 2011/10/04

3. El-Abhar HS, Schaalan MF. Phytotherapy in diabetes: review on potential mechanistic perspectives. World J Diabetes. 2014;5(2):176-97.. Epub 2014/04/22

4. Furukawa S, Fujita T, Shimabukuro M, Iwaki M, Yamada Y, Nakajima Y, et al. Increased oxidative stress in obesity and its impact on metabolic syndrome. J Clin Invest. 2004;114(12):1752-61.. Epub 2004/12/16

5. Demori I, Voci A, Fugassa E, Burlando B. Combined effects of high-fat diet and ethanol induce oxidative stress in rat liver. Alcohol. 2006;40(3):185-91. Epub 2007/04/10

6. Gomez-Perez Y, Amengual-Cladera E, Catala-Niell A, Thomas-Moya E, Gianotti M, Proenza AM, et al. Gender dimorphism in high-fat-diet-induced insulin resistance in skeletal muscle of aged rats. Cell Physiol Biochem: Int J Exp Cell Physiol Biochem Pharmacol. 2008;22(5-6):539-48.. Epub 2008/12/18

7. Vial G, Dubouchaud H, Couturier K, Cottet-Rousselle C, Taleux N, Athias A, et al. Effects of a high-fat diet on energy metabolism and ROS production in rat liver. J Hepatol. 2011:54(2):348-56. Epub 2010/11/27

8. El-Shiekh RA, Al-Mahdy DA, Mouneir SM, Hifnawy MS, Abdel-Sattar EA. Antiobesity effect of argel (Solenostemma argel) on obese rats fed a high fat diet. J Ethnopharmacol. 2019;111893. Epub 2019/04/19

9. Marshall JA, Bessesen DH. Dietary fat and the development of type 2 diabetes. Diabetes Care. 2002;25(3):620-2.. Epub 2002/03/05

10. Thanopoulou AC, Karamanos BG, Angelico FV, Assaad-Khalil SH, Barbato AF, Del Ben MP, et al. Dietary fat intake as risk factor for the development of diabetes: multinational, multicenter study of the Mediterranean Group for the Study of diabetes (MGSD). Diabetes Care. 2003;26(2):302-7.. Epub 2003/01/28

11. Roza NA, Possignolo LF, Palanch AC, Gontijo JA. Effect of long-term high-fat diet intake on peripheral insulin sensibility, blood pressure, and renal function in female rats. Food Nutr Res. 2016;60:28536.. Epub 2016/02/18
12. Steinberger J, Steffen L, Jacobs DR Jr, Moran A, Hong CP, Sinaiko AR. Relation of leptin to insulin resistance syndrome in children. Obes Res. 2003; 11(9):1124-30.. Epub 2003/09/16

13. Mohamed WS, Hassanien M, Sayed AK. Role of ghrelin, leptin and insulin resistance in development of metabolic syndrome in obese patients. Endocrinol Metab Synd. 2014;3(122):2161-1017.100012.

14. Khan W, Parveen R, Chester K, Parveen S, Ahmad S. Hypoglycemic potential of aqueous extract of Moringa oleifera leaf and in vivo GC-MS metabolomics. Front Pharmacol. 2017:8:577.. Epub 2017/09/29

15. Yassa HD, Tohamy AF. Extract of Moringa oleifera leaves ameliorates streptozotocin-induced diabetes mellitus in adult rats. Acta Histochem. 2014;116(5):844-54.. Epub 2014/03/25

16. Faroog F, Rai M, Tiwari A, Khan AA, Farooq S. Medicinal properties of Moringa oleifera: an overview of promising healer. J Med Plants Res. 2012; 6(27):4368-74.

17. Chuang PH, Lee CW, Chou JY, Murugan M, Shieh BJ, Chen HM. Anti-fungal activity of crude extracts and essential oil of Moringa oleifera lam. Bioresour Technol. 2007;98(1):232-6.. Epub 2006/01/13

18. Jaiswal D, Kumar Rai P, Kumar A, Mehta S, Watal G. Effect of Moringa oleifera lam. Leaves aqueous extract therapy on hyperglycemic rats. J Ethnopharmacol. 2009;123(3):392-6.. Epub 2009/06/09

19. Snarska A, Wysocka D, Rytel L. Effect of simvastatin on Thrombopoiesis in porcine bone marrow. J Vet Res. 2019;63(1):117-21.. Epub 2019/04/17

20. Hu XY, Ma YH, Wang C, Yang YH. Effects of simvastatin on cigarette smoke extract induced tissue-type plasminogen activator and plasminogen activator inhibitor-1 expression in human umbilical vein endothelial cells. Chin Med J. 2009;122(19):2380-5.. Epub 2010/01/19

21. Alshekhlee A, Katirji B. Clinical perspectives of statin-induced rhabdomyolysis. Am J Med. 2007;120(12):e29.. author reply e33. Epub 2007/12/07

22. Snarska A, Wysocka D, Rytel L, Gonkowski S, Pawelec H, Sobiech P. Simvastatin-induced changes in the Leukocytic system of porcine bone marrow. J Vet Res. 2018:62(3):329-33.. Epub 2018/12/26

23. Osman HM, Shayoub ME, Babiker EM. The effect of Moringa oleifera leaves on blood parameters and body weights of albino rats and rabbits. Jordan J Biol Sci. 2012;147(619):1-4.

24. Azza M, El-Habibi E, Mogalli A. Curcumin acts as cardiovascular protector via improving leptin and insulin resistance in obese male rats. J Am Sci. 2013; 9(3):397-405.

25. Singh Y, Garg MK, Tandon N, Marwaha RK. A study of insulin resistance by HOMA-IR and its cut-off value to identify metabolic syndrome in urban Indian adolescents. J Clin Res Pediatr Endocrinol. 2013;5(4):245-51.. Epub 2014/01/01

26. Chung A, Gurtu S, Chakravarthi S, Moorthy M, Palanisamy UD. Geraniin protects high-fat diet-induced oxidative stress in Sprague Dawley rats. Front Nutr. 2018:5:17.. Epub 2018/04/05

27. Ghasi S, Nwobodo E, Ofili J. Hypocholesterolemic effects of crude extract of leaf of Moringa oleifera lam in high-fat diet fed Wistar rats. J Ethnopharmacol. 2000;69(1):21-5

28. Ben Khedher MR, Hammami M, Arch JRS, Hislop DC, Eze D, Wargent ET, et al. Preventive effects of Salvia officinalis leaf extract on insulin resistance and inflammation in a model of high fat diet-induced obesity in mice that responds to rosiglitazone. PeerJ. 2018;6:e4166.. Epub 2018/01/16

29. Ashwini S, Bobby Z, Joseph M, Jacob SE, Padmapriya R. Insulin plant (Costus pictus) extract improves insulin sensitivity and ameliorates atherogenic dyslipidaemia in fructose induced insulin resistant rats: molecular mechanism. J Funct Foods. 2015;17:749-60.

30. Boden G, Chen X, Kolaczynski JW, Polansky M. Effects of prolonged hyperinsulinemia on serum leptin in normal human subjects. J Clin Invest. 1997;100(5):1107-13.. Epub 1997/09/01

31. Stefan N, Fritsche A, Haring H, Stumvoll M. Acute stimulation of leptin concentrations in humans during hyperglycemic hyperinsulinemia. Influence of free fatty acids and fasting. Int J Obesity Rel Metab Disord. 2001;25(1):138-42.. Epub 2001/03/13

32. Moonishaa TM, Nanda SK, Shamraj M, Sivaa R, Sivakumar P, Ravichandran K. Evaluation of leptin as a marker of insulin resistance in type 2 diabetes mellitus. Int J Appl Basic Med Res. 2017;7(3):176.

33. Kamegai J, Tamura H, Shimizu T, Ishii S, Sugihara H, Oikawa S. Effects of insulin, leptin, and glucagon on ghrelin secretion from isolated perfused rat stomach. Regul Pept. 2004;119(1-2):77-81.. Epub 2004/04/20

34. Shiiya $T$, Nakazato M, Mizuta M, Date $Y$, Mondal MS, Tanaka M, et al. Plasma ghrelin levels in lean and obese humans and the effect of 
glucose on ghrelin secretion. J Clin Endocrinol Metab. 2002;87(1):240-4.. Epub 2002/01/15

35. Lippl F, Erdmann J, Atmatzidis S, Schusdziarra V. Direct effect of leptin on gastric ghrelin secretion. Hormone Metab Res. 2005;37(2):123-5.. Epub 2005/03/22

36. Erdmann J, Lippl F, Wagenpfeil S, Schusdziarra V. Differential association of basal and postprandial plasma ghrelin with leptin, insulin, and type 2 diabetes. Diabetes. 2005;54(5):1371-8.. Epub 2005/04/28

37. Shimada M, Date Y, Mondal MS, Toshinai K, Shimbara T, Fukunaga K, et al. Somatostatin suppresses ghrelin secretion from the rat stomach. Biochem Biophys Res Commun. 2003;302(3):520-5.. Epub 2003/03/05

38. Zhang X, Chen W, Shao S, Xu G, Song Y, Xu C, et al. A high-fat diet rich in saturated and mono-unsaturated fatty acids induces disturbance of thyroid lipid profile and Hypothyroxinemia in male rats. Mol Nutr Food Res. 2018; 62(6):e1700599.. Epub 2018/01/25

39. Shao SS, Zhao YF, Song YF, Xu C, Yang JM, Xuan SM, et al. Dietary high-fat lard intake induces thyroid dysfunction and abnormal morphology in rats. Acta Pharmacol Sin. 2014;35(11):1411-20.. Epub 2014/09/30

40. Bensenor IM, Olmos RD, Lotufo PA. Hypothyroidism in the elderly: diagnosis and management. Clin Interv Aging. 2012;7:97.

41. Rahman HA, Sahib NG, Saari N, Abas F, Ismail A, Mumtaz MW, et al. Antiobesity effect of ethanolic extract from Cosmos caudatus Kunth leaf in lean rats fed a high fat diet. BMC Complement Altern Med. 2017;17(1):122.. Epub 2017/02/24

42. Matsuzawa-Nagata N, Takamura T, Ando H, Nakamura S, Kurita S, Misu H, et al. Increased oxidative stress precedes the onset of high-fat diet-induced insulin resistance and obesity. Metab Clin Exp. 2008;57(8):1071-7.. Epub 2008/07/22

43. Wannamethee SG, Tchernova J, Whincup P, Lowe GD, Kelly A, Rumley A, et al. Plasma leptin: associations with metabolic, inflammatory and haemostatic risk factors for cardiovascular disease. Atherosclerosis. 2007; 191(2):418-26.. Epub 2006/05/23

44. Huang CJ, McAllister MJ, Slusher AL, Webb HE, Mock JT, Acevedo EO. Obesity-related oxidative stress: the impact of physical activity and diet manipulation. Sports Med Open. 2015;1(1):32.. Epub 2015/10/06

45. Othman Al, El-Sawi MR, El-Missiry MA, Abukhalil MH. Epigallocatechin-3gallate protects against diabetic cardiomyopathy through modulating the cardiometabolic risk factors, oxidative stress, inflammation, cell death and fibrosis in streptozotocin-nicotinamide-induced diabetic rats. Biomed Pharmacother Biomed Pharmacother. 2017;94:362-73.. Epub 2017/08/05

46. Fridlyand $L E$, Philipson $L H$. Reactive species and early manifestation of insulin resistance in type 2 diabetes. Diabetes Obes Metab. 2006;8(2):13645.. Epub 2006/02/02

47. Meng R, Zhu DL, Bi Y, Yang DH, Wang YP. Anti-oxidative effect of apocynin on insulin resistance in high-fat diet mice. Ann Clin Lab Sci. 2011;41(3):23643.. Epub 2011/11/15

48. Maciejczyk M, Zebrowska E, Zalewska A, Chabowski A. Redox balance, antioxidant defense, and oxidative damage in the hypothalamus and cerebral cortex of rats with high fat diet-induced insulin resistance. Oxidative Med Cell Longev. 2018;2018:6940515.. Epub 2018/10/03

49. Pearson JK, Boyd RJ. Modeling the reduction of hydrogen peroxide by glutathione peroxidase mimics. J Phys Chem A. 2006;110(28):8979-85.. Epub 2006/07/14

50. El-Missiry MA, El Gindy AM. Amelioration of alloxan induced diabetes mellitus and oxidative stress in rats by oil of Eruca sativa seeds. Ann Nutr Metab. 2000:44(3):97-100.. Epub 2000/10/29

51. Soto CP, Perez BL, Favari LP, Reyes JL. Prevention of alloxan-induced diabetes mellitus in the rat by silymarin. Comp Biochem Physiol Part C: Pharmacol Toxicol Endocrinol. 1998;119(2):125-9.. Epub 1998/07/21

52. Razny U, Kiec-Wilk B, Wator L, Polus A, Dyduch G, Solnica B, et al. Increased nitric oxide availability attenuates high fat diet metabolic alterations and gene expression associated with insulin resistance. Cardiovasc Diabetol. 2011;10:68.. Epub 2011/07/26

53. Hon WM, Lee KH, Khoo HE. Nitric oxide in liver diseases. Ann N Y Acad Sci. 2002;962(1):275-95.

54. Beckman JS, Koppenol WH. Nitric oxide, superoxide, and peroxynitrite: the good, the bad, and ugly. Am J Phys. 1996;271(5 pt 1):C1424-37. Epub 1996/11/01

55. McConell GK, Wadley GD. Potential role of nitric oxide in contractionstimulated glucose uptake and mitochondrial biogenesis in skeletal muscle. Clin Exp Pharmacol Physiol. 2008;35(12):1488-92.. Epub 2008/09/02

\section{Submit your manuscript to a SpringerOpen ${ }^{\circ}$ journal and benefit from:}

- Convenient online submission

- Rigorous peer review

- Open access: articles freely available online

- High visibility within the field

- Retaining the copyright to your article

Submit your next manuscript at $\boldsymbol{\triangleright}$ springeropen.com 